\title{
A NOTE ON RECURRENT RANDOM WALKS
}

\author{
DIMITRIOS CHELIOTIS
}

\begin{abstract}
For any recurrent random walk $\left(S_{n}\right)_{n \geq 1}$ on $\mathbb{R}$, there are increasing sequences $\left(g_{n}\right)_{n \geq 1}$ converging to infinity for which $\left(g_{n} S_{n}\right)_{n \geq 1}$ has at least one finite accumulation point. For one class of random walks, we give a criterion on $\left(g_{n}\right)_{n \geq 1}$ and the distribution of $S_{1}$ determining the set of accumulation points for $\left(g_{n} S_{n}\right)_{n \geq 1}$. This extends, with a simpler proof, a result of K.L. Chung and P. Erdös. Finally, for recurrent, symmetric random walks, we give a criterion characterizing the increasing sequences $\left(g_{n}\right)_{n \geq 1}$ of positive numbers for which $\underline{\lim } g_{n}\left|S_{n}\right|=0$.
\end{abstract}

Keywords:random walk, recurrence, stable distributions, symmetric distributions.

\section{INTRODUCTION}

We recall that a random walk in $\mathbb{R}$ is any random sequence $\left(S_{n}\right)_{n \geq 1}$ with $S_{n}=X_{1}+\cdots+X_{n}$ and the $X_{i}$ 's i.i.d. having common distribution $F$. Such a walk is called recurrent if for any $\varepsilon>0$, with probability one, there are infinitely many indices $n$ for which $S_{n} \in(-\varepsilon, \varepsilon)$. It is easy to see that, for such a walk, it is possible to find a deterministic sequence of positive numbers $\left(\varepsilon_{n}\right)_{n \geq 1}$ converging to zero for which, with probability one, $S_{n} \in\left(-\varepsilon_{n}, \varepsilon_{n}\right)$ infinitely often. And in some cases it is possible to find a deterministic sequence of positive numbers $\left(\varepsilon_{n}\right)_{n \geq 1}$ converging to zero for which, with probability one, $S_{n} \notin\left(-\varepsilon_{n}, \varepsilon_{n}\right)$ eventually. This is the content of the next proposition, whose proof is given in Section 2

Proposition 1. Let $\left(Y_{n}\right)_{n \geq 1}$ be a sequence of random variables, defined on the same probability space, with $\underline{\lim }_{n \rightarrow+\infty}\left|Y_{n}\right|=0$ a.s. Then

(a) There exists a sequence of positive numbers $\left(g_{n}\right)_{n \geq 1}$ increasing to infinity with $\underline{\lim }_{n \rightarrow+\infty} g_{n}\left|Y_{n}\right|=0$ a.s.

(b) If $\sum_{n=1}^{+\infty} \mathbb{P}\left(Y_{n}=0\right)<+\infty$, then there exists a sequence of positive numbers $\left(g_{n}\right)_{n \geq 1}$ increasing to infinity with $\lim _{n \rightarrow+\infty} g_{n}\left|Y_{n}\right|=+\infty$ a.s.

By determining the set of sequences $\left(g_{n}\right)_{n \geq 1}$ with the properties $g_{n} \nearrow+\infty$ and $\underline{\lim }_{n \rightarrow+\infty} g_{n}\left|S_{n}\right|=0$, we measure in some way the "strength" of the recurrence of the random walk. We would like to establish a criterion characterizing these sequences in terms of the distribution function $F$.

Our first theorem addresses this question for the case where $F$ is the distribution of a symmetric random variable. In such a case, we call also $F$ symmetric.

Date: August 18, 2005.

Department of Mathematics, Bahen Center for Information Technology, 40 St. George St., 6th floor, Toronto, ON, M5S 3G3, Canada. E-mail address: dimitris@math.toronto.edu, URL: http://www.math.toronto.edu/dimitris/

Research partially supported by an anonymous Stanford Graduate Fellowship and a scholarship from the Alexander S. Onassis Public Benefit Foundation. 
Theorem 1. Assume that $F$ is symmetric. Then for any increasing sequence $\left(g_{n}\right)_{n \geq 1}$ of positive numbers and $M>0$,

$$
\underline{\lim } g_{n}\left|S_{n}\right|= \begin{cases}0 \text { a.s. } & \text { if } \sum_{n=1}^{+\infty} \mathbb{P}\left(g_{n}\left|S_{n}\right|<M\right)=+\infty \\ +\infty \text { a.s. } & \text { otherwise. }\end{cases}
$$

For the statement of our next theorem, we define for any sequence $x:=\left(x_{n}\right)_{n \geq 1}$ of real numbers

$$
K(x):=\left\{a \in \overline{\mathbb{R}}: a \text { is an accumulation point for }\left(x_{n}\right)_{n \geq 1}\right\},
$$

where $\overline{\mathbb{R}}:=\mathbb{R} \cup\{-\infty,+\infty\}$ is equipped with the usual topology. We also recall the following definition.

Definition 1. The distribution $F$ belongs to the domain of attraction of a distribution $R$ if there exist constants $a_{n}>0, b_{n}$ such that the distribution of $\left(S_{n}-b_{n}\right) / a_{n}$ tends to $R$ as $n \rightarrow+\infty$.

Theorem 2. Assume that $F$ belongs to the domain of attraction of a stable law, with zero centering constants (i.e., in the definition above, the $b_{n}$ 's are zero), and moreover that the characteristic function $\varphi$ of $F$ satisfies Cramer's condition $(C)$, i.e., $\varlimsup_{|t|}|++\infty| \varphi(t) \mid<1$. Then for any increasing sequence $\left(g_{n}\right)_{n \geq 1}$ of positive numbers, it holds

$$
K(g S)= \begin{cases}\{-\infty,+\infty\} & \text { a.s. if } \sum_{n=1}^{+\infty} \frac{1}{a_{n} g_{n}}<+\infty \\ \overline{\mathbb{R}} & \text { a.s. if } \sum_{n=1}^{+\infty} \frac{1}{a_{n} g_{n}}=+\infty\end{cases}
$$

In the case where $F$ itself is the distribution of a symmetric stable law with index in $[1,2]$, we have the following corollary.

Corollary 1. For a symmetric stable law with index $\alpha \in[1,2]$ (i.e., characteristic function $\varphi(t)=e^{-c|t|^{\alpha}}$ for some $c>0$ ),

$$
K\left(\left(n^{\varepsilon} S_{n}\right)_{n \geq 1}\right)= \begin{cases}\overline{\mathbb{R}} & \text { a.s. if } \varepsilon \leq 1-\frac{1}{\alpha}, \\ \{-\infty,+\infty\} & \text { a.s. if } \varepsilon>1-\frac{1}{\alpha} .\end{cases}
$$

Remark 1. When $g_{n}=1$ for all $n$, Theorem 1 reduces to a well known criterion for recurrence, which holds for all distributions (see Durrett (1996), Chapter 3, Corollary 2.6)

Remark 2. If $\left(S_{n}\right)_{n \geq 1}$ is a recurrent random walk and $\left(g_{n}\right)_{n \geq 1}$ is an increasing sequence of positive numbers, then $-\infty,+\infty \in K(g S)$ because $\underline{\lim }_{n \rightarrow+\infty} S_{n}=-\infty, \varlimsup_{n \rightarrow+\infty} S_{n}=+\infty$ (see Durrett (1996)), Chapter 3, Theorem 1.2). So the above theorems, in particular, characterize the sequences $\left(g_{n}\right)_{n \geq 1}$ for which $K(g S)=$ $\{-\infty,+\infty\}$, i.e., the ones that push the walk to infinity.

Remark 3. Let $\varphi$ denote the characteristic function corresponding to the distribution $F$. If $F$ is lattice with span $h>0$ and the corresponding walk recurrent, then $\mathbb{P}\left(S_{n}=k h\right.$ i.o. $)=1$ for all $k \in \mathbb{Z}$, and $K(g S)=\{0\} \cup\left\{k h \lim _{n \rightarrow+\infty} g_{n}: k \in \mathbb{Z} \backslash\{0\}\right\}$ for all increasing sequences $\left(g_{n}\right)_{n \geq 1}$ of positive numbers. In particular, $K(g S) \neq\{-\infty,+\infty\}$ for all such sequences; i.e., the walk is very recurrent but for a trivial reason. That is, because $\mathbb{P}\left(S_{n}=0\right.$ i.o. $)=1$. The remaining distributions, called nonlattice, are the ones satisfying $|\varphi(t)|<1$ for all $t \in \mathbb{R} \backslash\{0\}$. A subclass of these are those that satisfy Cramer's condition (C), and Theorem 2 is concerned with these. In that theorem, the assumption that $F$ satisfies Cramer's condition (C) cannot be 
weakened to $F$ nonlattice. To see this, take $a \in \mathbb{R} \backslash \mathbb{Q}$ and the distribution $F$ that assigns mass $1 / 4$ to each of the numbers in the set $\{-1,1,-a, a\}$. $F$ belongs to the domain of attraction of the normal distribution, with $a_{n}=\sqrt{n}$ and zero centering constants. Its characteristic function is $\varphi(t)=(\cos t+\cos a t) / 2$ for all $t \in \mathbb{R}$ and satisfies $|\varphi(t)|<1$ for all $t \in \mathbb{R} \backslash\{0\}$ since $a$ is irrational. Thus, the distribution is nonlattice. But it does not satisfy Cramer's condition (C) because $\varlimsup_{k \rightarrow+\infty, k \in \mathbb{N}}(a k-[a k])=1$ due again to the irrationality of $a$; and $\varphi(2 k \pi)=(1+\cos ((a k-[a k]) 2 \pi)) / 2$ for $k \in \mathbb{N}$. For the sequence $(n)_{n \geq 1}$ we have $\sum_{n=1}^{+\infty}(\sqrt{n} n)^{-1}<+\infty$. If our theorem would apply to this case, we would have $\lim n\left|S_{n}\right|=+\infty$ a.s. This is false, because for $n \geq 1$ we have $\mathbb{P}\left(S_{2 n}=0\right)=4^{-2 n} \sum_{m=0}^{n}(2 n) ! /[m ! m !(n-m) !(n-m) !] \sim(\pi n)^{-1}$ (it is the same calculation as for the two dimensional simple random walk since $a$ is irrational), implying $\sum_{n=1}^{+\infty} \mathbb{P}\left(S_{2 n}=0\right)=+\infty$, and by a well known result, $\mathbb{P}\left(S_{2 n}=0\right.$ i.o. $)=1$ (see Durrett (1996), Chapter 3, Theorem 2.2).

The first result in the spirit of our theorems is the content of Theorem 3 of Chung and Erdos (1947), where the authors characterize the sequences $\left(g_{n}\right)_{n \geq 1}$ for which $\left(g_{n} / \sqrt{n}\right)_{n \geq 1}$ increases to infinity and $\underline{\lim }_{n \rightarrow+\infty} g_{n}\left|S_{n}\right|=0$. They assume that the distribution of the $X_{i}$ 's has finite absolute fifth moment, zero mean value, and a non zero absolutely continuous part. Later, V.V.Petrov (see Petrov (1979)) improved their result by assuming only finite second moment, zero expectation, and Cramer's condition stated above; also he removed the requirement that $\left(g_{n} / \sqrt{n}\right)_{n \geq 1}$ converges to infinity and assumed that it is just increasing.

Our second result is an extension of the work of Erdos, Chung, Petrov as it determines all accumulation points of $\left(g_{n} S_{n}\right)_{n \geq 1}$ for a bigger class of distributions $F$ and sequences $\left(g_{n}\right)_{n \geq 1}$. The proofs of the aforementioned authors are longer than ours because they establish from first principles that a set of interest has probability one. In our approach, we just prove that this set has positive probability, and then we invoke the Hewitt-Savage zero-one law.

Finally, we should mention the following result of V. Petrov (Theorem 6.21 in Petrov (1995)). Assume that the characteristic function of the $X_{i}$ 's satisfies Cramer's condition (C). Then for any sequence $\left(g_{n}\right)_{n \geq 1}$ of positive numbers, $\sum_{n=1}^{+\infty}\left(\sqrt{n} g_{n}\right)^{-1}<+\infty$ implies $\lim _{n \rightarrow+\infty} g_{n}\left|S_{n}\right|=+\infty$ a.s.

\section{Proof of the RESUlts}

In this section, we prove the two theorems and Proposition 1 First, we give a version of the second Borel-Cantelli lemma that we will use. It is a simple application of the Kochen-Stone lemma (see Durrett (1996), Chapter 1, exercise 6.20), so we omit its proof.

Lemma 1. Assume that $\left(A_{n}\right)_{n \geq 1}$ is a sequence of measurable sets and that there are $n_{0} \geq 1$ and $c>0$ such that $\mathbb{P}\left(A_{j} \cap A_{k}\right) \leq c \mathbb{P}\left(A_{j}\right) \mathbb{P}\left(A_{k-j}\right)$ for every $k, j \geq n_{0}$ with $k-j \geq n_{0}$. Then

$$
\mathbb{P}\left(A_{n} \text { i.o. }\right)>0 \quad \text { iff } \quad \sum_{n=0}^{+\infty} \mathbb{P}\left(A_{n}\right)=+\infty .
$$

For $a \in \mathbb{R}, \varepsilon>0$, and $n \geq 1$, we set $A_{n}(a, \varepsilon):=\left[g_{n} S_{n} \in(a-\varepsilon, a+\varepsilon)\right]$, and observe that

$$
g_{n} S_{n} \in(a-\varepsilon, a+\varepsilon) \text { i.o. a.s. } \Leftrightarrow \mathbb{P}\left(\varlimsup_{n} A_{n}(a, \varepsilon)\right)=1 .
$$

Since the $X_{i}$ 's are i.i.d and $\varlimsup_{n} A_{n}(a, \varepsilon)$ is an exchangeable event, by the Hewitt-Savage zero-one law, if it has positive measure, then it has measure one. Both theorems (1) and 2) are proved by showing that the 
sequence $\left(A_{n}(a, \varepsilon)\right)_{n \geq 1}$ satisfies the conditions of Lemma 1 for all $\varepsilon>0$ and appropriate $a$ (for $a=0$ for Theorem 1] and for all $a \in \mathbb{R}$ for Theorem 2).

To do this, we observe that for $a \in \mathbb{R}, \varepsilon>0$, and any $j<k$,

$$
\begin{aligned}
& \mathbb{P}\left(A_{j}(a, \varepsilon) \cap A_{k}(a, \varepsilon)\right) \\
& \quad=\mathbb{P}\left(g_{j} S_{j} \in(a-\varepsilon, a+\varepsilon), g_{k}\left(S_{k}-S_{j}\right) \in\left(-g_{k} S_{j}+a-\varepsilon,-g_{k} S_{j}+a+\varepsilon\right)\right) \\
& \quad \leq \mathbb{P}\left(A_{j}(a, \varepsilon)\right) \sup _{y \in\left((a-\varepsilon) / g_{j},(a+\varepsilon) / g_{j}\right)} \mathbb{P}\left(S_{k-j} \in\left(-y+\frac{a-\varepsilon}{g_{k}},-y+\frac{a+\varepsilon}{g_{k}}\right)\right) .
\end{aligned}
$$

And now we want to bound the last supremum by $c \mathbb{P}\left(A_{k-j}(a, \varepsilon)\right)$ for some constant $c$ that may depend on $a, \varepsilon$ but not on $k, j$. It remains to establish such a bound under the assumptions of either of the two theorems.

Theorem 1 is a consequence of the following two results.

Proposition 2. Assume that $F$ is symmetric. Then for any increasing sequence $\left(g_{n}\right)_{n \geq 1}$ of positive numbers and $M>0$,

$$
\mathbb{P}\left(g_{n}\left|S_{n}\right|<M \text { i.o. }\right)= \begin{cases}1 & \text { if } \sum_{n=1}^{+\infty} \mathbb{P}\left(g_{n}\left|S_{n}\right|<M\right)=+\infty \\ 0 & \text { otherwise. }\end{cases}
$$

Proof. If $\sum_{n=1}^{+\infty} \mathbb{P}\left(g_{n}\left|S_{n}\right|<M\right)<\infty$, we apply the first Borel-Cantelli lemma. So assume $\sum_{n=1}^{+\infty} \mathbb{P}\left(g_{n}\left|S_{n}\right|<M\right)=+\infty$. We claim that $\sum_{n=1}^{+\infty} \mathbb{P}\left(g_{2 n}\left|S_{2 n}\right|<M\right)=+\infty$. Indeed, for $n \geq 1$ we have

$$
\begin{array}{r}
\mathbb{P}\left(\left|S_{2 n+1}\right|<M / g_{2 n+1}\right)=\int \mathbb{P}\left(\left|S_{2 n}+y\right|<M / g_{2 n+1}\right) d F_{X}(y) \\
\quad \leq 4 \mathbb{P}\left(\left|S_{2 n}\right|<M / g_{2 n+1}\right) \leq 4 \mathbb{P}\left(\left|S_{2 n}\right|<M / g_{2 n}\right) .
\end{array}
$$

The first inequality follows from Lemma 3 because the symmetry of the distribution of the $X_{i}$ 's implies that $S_{2 n}$ has a nonnegative characteristic function. The second inequality follows from the monotonicity of $\left(g_{n}\right)_{n \geq 1}$.

Combining $\sum_{n=1}^{+\infty} \mathbb{P}\left(g_{2 n}\left|S_{2 n}\right|<M\right)=+\infty$ with the comments before the proof of this proposition (applied to the random walk $\left(S_{2 n}\right)_{n \geq 1}$ and the sequence $\left.\left(g_{2 n}\right)_{n \geq 1}\right)$ and Lemma 3 we get the desired result.

Lemma 2. Assume that $F$ is symmetric and $\left(g_{n}\right)_{n \geq 1}$ is an increasing sequence of positive numbers. Then $\sum_{n=1}^{+\infty} \mathbb{P}\left(g_{n}\left|S_{n}\right|<M\right)$ is either infinite for all $M>0$ or finite for all $M>0$.

Proof. Assume the contrary. Then there is an $a>0$ so that the above sum is finite for $M<a$ and infinite for $M>a$. Take $\varepsilon \in(0, a)$. Since

$$
\mathbb{P}\left(g_{n}\left|S_{n}\right|<a+\varepsilon\right)=\mathbb{P}\left(g_{n}\left|S_{n}\right| \leq a-\varepsilon\right)+\mathbb{P}\left(g_{n}\left|S_{n}\right| \in(a-\varepsilon, a+\varepsilon)\right),
$$

it follows that

$$
\sum_{n=1}^{+\infty} \mathbb{P}\left(g_{n}\left|S_{n}\right| \in(a-\varepsilon, a+\varepsilon)\right)=+\infty
$$


As in the proof of Proposition 2 we will show that $\sum_{n=1}^{+\infty} \mathbb{P}\left(g_{2 n}\left|S_{2 n}\right|<\varepsilon\right)=+\infty$. Indeed, for $n \geq 1$,

$$
\begin{aligned}
\mathbb{P}\left(g_{2 n+1}\left|S_{2 n+1}\right| \in(a-\varepsilon, a+\varepsilon)\right) & \\
=\int \mathbb{P}\left(S_{2 n} \in\left(-x+\frac{a-\varepsilon}{g_{2 n+1}},-x+\frac{a+\varepsilon}{g_{2 n+1}}\right) \cup\left(-x-\frac{a+\varepsilon}{g_{2 n+1}},-x-\frac{a-\varepsilon}{g_{2 n+1}}\right)\right) d F(x) & \leq 8 \mathbb{P}\left(S_{2 n} \in\left(-\frac{\varepsilon}{g_{2 n+1}}, \frac{\varepsilon}{g_{2 n+1}}\right)\right) \leq 8 \mathbb{P}\left(S_{2 n} \in\left(-\frac{\varepsilon}{g_{2 n}}, \frac{\varepsilon}{g_{2 n}}\right)\right) .
\end{aligned}
$$

Also $\mathbb{P}\left(g_{2 n}\left|S_{2 n}\right| \in(a-\varepsilon, a+\varepsilon)\right) \leq 8 \mathbb{P}\left(g_{2 n}\left|S_{2 n}\right|<\varepsilon\right)$. Thus, (1) and the last two inequalities imply $\sum_{n=1}^{+\infty} \mathbb{P}\left(g_{2 n}\left|S_{2 n}\right|<\varepsilon\right)=+\infty$. A contradiction since $\varepsilon<a$.

The essential ingredient for the proof of Proposition 2 and Lemma 2 is the following lemma.

Lemma 3. If $X$ is a random variable with real and nonnegative characteristic function $\varphi$, then for all $x \in \mathbb{R}$ and $\delta>0$ we have

$$
\mathbb{P}(X \in[x-\delta, x+\delta]) \leq 4 \mathbb{P}(X \in(-\delta, \delta)) .
$$

Proof. Clearly, we can assume that $\delta=1$. Let $A: \mathbb{R} \rightarrow \mathbb{R}$ be defined by $A(x)=\int_{0}^{1} \mathbb{P}(X \in[x-r, x+r]) d r$ for every $x \in \mathbb{R}$. Then using the inversion formula and the bounded convergence theorem, we get $A(x)=$ $(2 \pi)^{-1} \int_{\mathbb{R}} e^{-i t x}(1-\cos t) t^{-2} \varphi(t) d t$, which attains global maximum at $x=0$ because $\varphi$ is nonnegative. Thus $\mathbb{P}(X \in(-1,1)) \geq A(0) \geq A(x) \geq \mathbb{P}(X \in[x-1 / 2, x+1 / 2]) / 2$. Consequently $\mathbb{P}(X \in[x-1, x+1]) \leq 4 \mathbb{P}(X \in$ $(-1,1))$.

For the proof of Theorem 2] we will use the following two facts.

Fact 1. Let $S_{n}, a_{n}, b_{n}$ be as in Definition 1, with the law of $\left(S_{n}-b_{n}\right) / a_{n}$ converging to a stable distribution with index $\alpha$, not concentrated at zero. Then $h(n):=a_{n} / n^{1 / a}$ is a function slowly varying at infinity.

Fact 2. If $h$ is a function slowly varying at infinity and $\delta>0$, then $n^{-\delta}<h(n)<n^{\delta}$ for all big $n$.

Fact 1 is contained in Theorem 2.1.1 of Ibragimov and Linnik (1971). Fact 2 is Lemma 2 of $\S 8$, Chapter VIII in Feller (1971).

\section{Proof of Theorem 2,}

Call $F_{\alpha}$ the distribution function of the limiting stable law. Its characteristic function is absolutely integrable (see Feller (1971), Chapter XVII, Section 6), so it has a continuous bounded density, call it $f_{\alpha}$. We will consider two cases depending on the value of $\alpha$. The nontrivial is the second one.

CASE 1: $0<\alpha<1$.

By Facts 1,2 , we have $\sum_{n=1}^{+\infty} 1 / a_{n}<+\infty$, so the series in the statement of the theorem converges always. It is enough to prove that $S_{n}$ is transient. Let $M$ be the bound in the density of $f_{\alpha}$. By Lemma 2 in Stone (1965), there exists an $n_{0} \geq 1$ and $h_{0}>0$ such that for all $x \in \mathbb{R}$ and $h \in\left[a_{n}^{-1}, h_{0}\right]$ we have

$$
h\left(f_{\alpha}(x)-1\right) \leq \mathbb{P}\left(S_{n} / a_{n} \in[x, x+h]\right) \leq h\left(f_{\alpha}(x)-1\right) .
$$

To apply that lemma one needs to have $F$ nonlattice, which is true because $\varphi$ satisfies Cramer's condition (C). Now pick $\varepsilon \in(\alpha, 1)$. Then for $h:=2 a_{n}^{-\varepsilon}$ and $x:=-h / 2$, we have $\mathbb{P}\left(\left|S_{n}\right|<a_{n}^{1-\varepsilon}\right)=\mathbb{P}\left(\left|S_{n} / a_{n}\right|<a_{n}^{-\varepsilon}\right) \leq$ 
$(M+1) a_{n}^{-\varepsilon}$ for big $n$. Since $\varepsilon / \alpha>1$, Facts 1,2 imply that $\sum_{n=1}^{+\infty} a_{n}^{-\varepsilon}<+\infty$, and the first Borel-Cantelli lemma gives that $\left|S_{n}\right| \geq a_{n}^{1-\varepsilon}$ eventually. So the walk is transient since $1-\varepsilon>0$.

CASE 2: $1 \leq \alpha \leq 2$.

Assume that $\overline{\lim }_{|t| \rightarrow+\infty}|\varphi(t)|=\theta<1$, and pick an $\varepsilon_{0}>0$ so that $\theta+\varepsilon_{0}<1$.

Step 1: First we prove the theorem for all sequences $\left(g_{n}\right)_{n \geq 1}$ as in the statement of the theorem that moreover satisfy

$$
\lim _{n \rightarrow+\infty} g_{n} a_{n}\left(\theta+\varepsilon_{0}\right)^{n}=0 .
$$

Using the explicit expressions for $f_{\alpha}$ (see Lemma 1 in Fellen (1971), Chapter XVII, Section 6), we can see that $f_{\alpha}(0)>0$ for $\alpha \in(1,2]$. For $\alpha=1$, we use the fact that $F_{\alpha}$ is strictly stable (i.e. the centering constants are zero) to obtain that its characteristic function is of the form $\varphi_{1}(t)=e^{i t c-d|t|}$ for all $t \in \mathbb{R}$, where $c \in \mathbb{R}$ and $d>0$ are some constants (e.g., by exploiting the relation $\varphi_{1}(n t)=\left(\varphi_{1}(t)\right)^{n}$ for all $n \in \mathbb{N}$ and $t \in \mathbb{R}$, and the general form of the characteristic function of a stable distribution with index 1 given in Theorem 9.32 of Breiman (1992)). Thus, $F_{1}$ is the distribution function of $Y d+c$, where $Y$ has a Cauchy distribution, which implies that $f_{1}(0)>0$.

By the Local Limit Theorem of Stone (see Stone (1965)), for fixed $c>1$, there is an $h_{0}>0$ and $n_{0}>1$ such that for any interval $I \subset\left[-h_{0}, h_{0}\right]$ and $n \geq n_{0}$ with $|I|>\left(\theta+\varepsilon_{0}\right)^{n}$, we have

$$
\frac{1}{c} f_{\alpha}(0)<\frac{1}{|I|} \mathbb{P}\left(\frac{S_{n}}{a_{n}} \in I\right)<c f_{\alpha}(0) .
$$

This theorem applies because $\varphi$ satisfies Cramer's condition (C).

Now take $a \in \mathbb{R}$ and $\varepsilon>0$. Since $g_{n} a_{n}\left(\theta+\varepsilon_{0}\right)^{n} \rightarrow 0$ and $a_{n} g_{n} \rightarrow+\infty$ as $n \rightarrow+\infty$ (see Facts 1,2 regarding $\left.a_{n}\right)$, we can assume that for the above $n_{0}$ we also have $(|a|+\varepsilon) / a_{n} g_{n}<h_{0} / 2, g_{n} a_{n}\left(\theta+\varepsilon_{0}\right)^{n} / 2 \varepsilon<1$ for all $n \geq n_{0}$. Thus, for $n \geq n_{0}$ and $I:=\left((a-\varepsilon) / a_{n} g_{n},(a+\varepsilon) / a_{n} g_{n}\right)$, (3) applies and gives

$$
c^{-1} f_{\alpha}(0)<\frac{a_{n} g_{n}}{2 \varepsilon} \mathbb{P}\left(A_{n}(a, \varepsilon)\right)<c f_{\alpha}(0) .
$$

Also

$$
\begin{aligned}
\mathbb{P}\left(S _ { k - j } \in \left(-y+\frac{a-\varepsilon}{g_{k}},\right.\right. & \left.\left.-y+\frac{a+\varepsilon}{g_{k}}\right)\right) \\
= & \mathbb{P}\left(\frac{S_{k-j}}{a_{k-j}} \in\left(-\frac{y}{a_{k-j}}+\frac{a-\varepsilon}{g_{k} a_{k-j}},-\frac{y}{a_{k-j}}+\frac{a+\varepsilon}{g_{k} a_{k-j}}\right)\right) \\
\leq & \mathbb{P}\left(\frac{S_{k-j}}{a_{k-j}} \in\left(-\frac{y}{a_{k-j}}+\frac{a}{g_{k} a_{k-j}}-\frac{\varepsilon}{g_{k-j} a_{k-j}},-\frac{y}{a_{k-j}}+\frac{a}{g_{k} a_{k-j}}+\frac{\varepsilon}{g_{k-j} a_{k-j}}\right)\right),
\end{aligned}
$$

where in the last inequality we used the monotonicity of $g$. Again (3) applies, and in conjunction with (4) gives

$$
\sup _{y \in\left((a-\varepsilon) / g_{j},(a+\varepsilon) / g_{j}\right)} \mathbb{P}\left(S_{k-j} \in\left(-y+\frac{a-\varepsilon}{g_{k}},-y+\frac{a+\varepsilon}{g_{k}}\right)\right)
$$

$$
<c f_{\alpha}(0) \frac{2 \varepsilon}{a_{k-j} g_{k-j}}<c^{2} \mathbb{P}\left(A_{k-j}(a, \varepsilon)\right)
$$

for $k, j \geq n_{0}$ with $k-j \geq n_{0}$. Relation (4) implies that for every $\varepsilon>0$ the two series $\sum_{n=0}^{+\infty} \mathbb{P}\left(A_{n}(a, \varepsilon)\right)$ and $\sum_{n=1}^{+\infty}\left(a_{n} g_{n}\right)^{-1}$ converge or diverge together. When $\sum_{n=1}^{+\infty}\left(a_{n} g_{n}\right)^{-1}<\infty$, the first Borel-Cantelli lemma 
applied to the sequence of the $A_{n}(a, \varepsilon)$ 's with the choices $a=0, \varepsilon=k, k \in \mathbb{N}$, gives $\lim _{n \rightarrow+\infty} g_{n}\left|S_{n}\right|=+\infty$ (i.e., there are no finite accumulation points). When this series diverges, the result follows on applying Lemma 1 to the $A_{n}(a, \varepsilon)$ 's with the choices $a \in \mathbb{Q}, \varepsilon=1 / k, k \in \mathbb{N} \backslash\{0\}$ and observing that $K(g S)$ is a closed subset of $\overline{\mathbb{R}}$.

Step 2: For the sequence $g_{n}:=n$, using Facts 1,2, we see that (2) holds and $\sum_{n=1}^{+\infty} \frac{1}{a_{n} g_{n}}<+\infty$. Consequently, by Step $1, K(g S)=\{-\infty,+\infty\}$.

Step 3: Now let $\left(g_{n}\right)_{n \geq 1}$ be any increasing sequence of positive numbers. Introduce $g_{n}^{\prime}=\min \left\{g_{n}, n\right\}$ for $n \geq 1$. Then $\left(g_{n}^{\prime}\right)_{n \geq 1}$ satisfies $\lim _{n \rightarrow+\infty} g_{n}^{\prime} a_{n}(\theta+\varepsilon)^{n}=0$.

If $\sum_{n=1}^{+\infty}\left(a_{n} g_{n}\right)^{-1}<\infty$, then since $1 / g_{n}^{\prime}<1 / g_{n}+1 / n$ and $\sum_{n=1}^{+\infty}\left(a_{n} n\right)^{-1}<\infty$, it follows that $\sum_{n=1}^{+\infty}\left(a_{n} g_{n}^{\prime}\right)^{-1}<$ $\infty$. By the result of Step 1, it follows that $\underline{\lim }_{n \rightarrow+\infty} g_{n}^{\prime}\left|S_{n}\right|=+\infty$. Combining this with $g_{n}^{\prime} \leq g_{n}$, we get $\underline{\lim }_{n \rightarrow+\infty} g_{n}\left|S_{n}\right|=+\infty$.

If $\sum_{n=1}^{+\infty}\left(a_{n} g_{n}\right)^{-1}=+\infty$, then $g_{n}^{\prime} \leq g_{n}$ implies that $\sum_{n=1}^{+\infty}\left(a_{n} g_{n}^{\prime}\right)^{-1}=+\infty$. By Step $1, K\left(g^{\prime} S\right)=\overline{\mathbb{R}}$; and since $\lim _{n \rightarrow+\infty} n\left|S_{n}\right|=+\infty$ (Step 2), we conclude that $K(g S)=\overline{\mathbb{R}}$.

\section{Proof of Proposition 1 .}

(a) We set $A_{n}(\varepsilon):=\left[\left|Y_{n}\right|<\varepsilon\right]$ for all $n \in \mathbb{N} \backslash\{0\}$ and $\varepsilon>0$. By the assumption, $\mathbb{P}\left(\cup_{k=n}^{+\infty} A_{k}(\varepsilon)\right)=1$ for all $\varepsilon>0$ and $n \geq 1$. Recursively we construct a strictly increasing sequence of positive integers $\left(n_{j}\right)_{j \geq 0}$ with $n_{0}=1$ and

$$
\mathbb{P}\left(\cup_{k=n_{j-1}}^{n_{j}-1} A_{k}\left(\frac{1}{j}\right)\right) \geq 1-\frac{1}{2^{j}} .
$$

Define $g_{n}=\sqrt{j}$ for $n_{j-1} \leq n<n_{j}$. By (6) and the first Borel-Cantelli lemma, we have that with probability one there is a random $j_{0}$ so that for all $j \geq j_{0}$ there is a $k_{j} \in\left[n_{j-1}, n_{j}-1\right]$ with $\left|Y_{k_{j}}\right|<1 / j$; i.e., $g_{k_{j}}\left|Y_{k_{j}}\right|<1 / \sqrt{j}$. This proves (a).

(b) For every $n \geq 1$, there is a $\delta_{n} \in(0,1)$ so that $\mathbb{P}\left(\left|Y_{n}\right|<\delta_{n}\right) \leq 2 \max \left\{P\left(Y_{n}=0\right), 2^{-n}\right\}$. Let $h_{n}:=n / \delta_{n}$ for all $n \geq 1$. Then $\sum_{n=1}^{+\infty} \mathbb{P}\left(h_{n}\left|Y_{n}\right|<n\right)<+\infty$, which implies that $\lim _{n \rightarrow+\infty} h_{n}\left|Y_{n}\right|=+\infty$ a.s. Let $g_{n}=\max \left\{h_{1}, h_{2}, \cdots, h_{n}\right\}$.

Proposition 1(a) says that all recurrent sequences $\left(Y_{n}\right)_{n \geq 1}$ can afford to get increased and still be recurrent. There is no recurrent sequence that is "on the brink of loosing its recurrence."

\section{The Multidimensional CASE}

Again we consider a sequence $\left(X_{i}\right)_{i \geq 1}$ of i.i.d random variables with values in $\mathbb{R}^{d}$ with $d \geq 2$ and the corresponding random walk $\left(S_{n}\right)_{n \geq 1}$ with $S_{n}=X_{1}+\cdots+X_{n}$. If the support of the distribution of $X_{1}$ is not contained in a two dimensional subspace of $\mathbb{R}^{d}$, then the walk is transient (see Durrett (1996). Chapter 3 , Theorem 2.12). So the only meaningful multidimensional case is when $d=2$. Our results, concerning random walks in $\mathbb{R}$, have analogs in this case too. Now for a sequence $x:=\left(x_{n}\right)_{n \geq 1}$ in $\mathbb{R}^{2}$, we define

$$
K(x):=\left\{a \in \mathbb{R}^{2} \cup\{\infty\}: a \text { is an accumulation point for }\left(x_{n}\right)_{n \geq 1}\right\}
$$

where $\mathbb{R}^{2} \cup\{\infty\}$ is the one point compactification of $\mathbb{R}^{2}$ equipped with the usual topology. In this topology, $K(x)$ is a closed set.

The analog of Theorem 1 holds in dimension two also. We only need in its statement to write $\left\|S_{n}\right\|_{\infty}$ instead of $\left|S_{n}\right|$ (of course any other equivalent norm in $\mathbb{R}^{2}$ works). The crucial element in its proof is again 
the analog of Lemma 3 which reads $\mathbb{P}\left(\|X-x\|_{\infty} \leq \delta\right) \leq 16 \mathbb{P}\left(\|X\|_{\infty}<\delta\right)$ for any two dimensional random variable $X$ with nonnegative characteristic function, and any $x \in \mathbb{R}^{2}, \delta>0$. The proof is done in the same way as that of Lemma B] using the function $A\left(\left(x_{1}, x_{2}\right)\right):=\int_{[0,1] \times[0,1]} \mathbb{P}\left(\left|X_{1}-x_{1}\right|<r_{1},\left|X_{2}-x_{2}\right|<r_{2}\right) d r_{1} d r_{2}$, where $X=\left(X_{1}, X_{2}\right)$.

For the analog of Theorem [2] we assume that $F$, the common distribution function of the $X_{i}$ 's, has support not contained on a proper subspace of $\mathbb{R}^{2}$. Also that it belongs to the domain of attraction of a stable distribution $F_{\alpha}$ with index $\alpha \in(0,2]$, with zero centering constants, and that the characteristic function $\varphi$ of $F$ (defined by $\varphi(t):=\int_{\mathbb{R}} e^{i t \cdot x} d F(x)$ for all $t \in \mathbb{R}^{2}$ ) satisfies Cramer's condition (C); that is, $\varlimsup_{|t| \rightarrow+\infty}|\varphi(t)|<1$. Then it can be seen, using the local limit theorem of Stone and well known criteria for recurrence (see, e.g., Durrett (1996), Chapter 3, Lemma 2.4), that the walk is recurrent only when $\alpha=2$. The next theorem concerns this case.

Theorem 3. Assume that $F$ belongs to the domain of attraction of a non-degenerate two dimensional normal distribution, with zero centering constants, and moreover that the characteristic function of $F$ satisfies Cramer's condition $(C)$. Then for any increasing sequence $\left(g_{n}\right)_{n \geq 1}$ of positive numbers, it holds

$$
K(g S)= \begin{cases}\{\infty\} & \text { a.s. if } \sum_{n=1}^{+\infty} \frac{1}{\left(a_{n} g_{n}\right)^{2}}<+\infty, \\ \mathbb{R}^{2} \cup\{\infty\} & \text { a.s. if } \sum_{n=1}^{+\infty} \frac{1}{\left(a_{n} g_{n}\right)^{2}}=+\infty .\end{cases}
$$

The proof goes exactly as that of Theorem 2

Acknowledgement. I am grateful to Bálint Virág for showing me a proof of Lemma 3 before I got the one included now. Also for comments that improved the presentation of the paper.

\section{REFERENCES}

Breiman, L. (1992). Probability, Society for Industrial and Applied Mathematics, Philadelphia, PA.

Chung, K.L. and Erdos, P. (1947). On the lower limit of sums of independent random variables, Ann. Math.

48, no. 4: 1003-1013.

Durrett, R. (1996). Probability: Theory and examples, Wadsworth Pub. Co, second edn.

Feller, W. (1971). An introduction to probability theory and its applications, vol. 2, John Wiley and Sons, Inc., New York-London-Sydney, second edn.

Ibragimov, I. A. and Linnik, Yu. V. (1971). Independent and stationary sequences of random variables. With a supplementary chapter by I. A. Ibragimov and V. V. Petrov. Translation from the Russian edited by J. F. C. Kingman, Wolters-Noordhoff Publishing, Groningen.

Petrov, V. V. (1979). Remark on the lower limit for the modulus of sums of independent random variables, Lithuanian Math. J. 18, no. 4: 528-531.

Petrov, V. V. (1995). Limit theorems of probability theory. Sequences of independent random variables, Oxford University Press, New York.

Stone, C. (1965). A local limit theorem for non-lattice multidimensional distribution functions, Ann. Math.

Statist 36: $546-551$. 